\title{
Generalized Performance Characteristics of Refrigeration and Heat Pump Systems
}

\author{
Mahmoud Huleihil $^{1}$ and Bjarne Andresen ${ }^{2}$ \\ ${ }^{1}$ Academic Institute for Training Arab Teachers (AITAT), Beit Berl College, Doar Beit Berl 44905, Israel \\ ${ }^{2}$ Niels Bohr Institute, University of Copenhagen, Universitetsparken 5, DK-2100 Copenhagen Ø, Denmark \\ Correspondence should be addressed to Mahmoud Huleihil, cs.berl@gmail.com
}

Received 19 September 2010; Accepted 1 November 2010

Academic Editor: Steven Sherwood

Copyright ( 2010 M. Huleihil and B. Andresen. This is an open access article distributed under the Creative Commons Attribution License, which permits unrestricted use, distribution, and reproduction in any medium, provided the original work is properly cited.

\begin{abstract}
A finite-time generic model to describe the behavior of real refrigeration systems is discussed. The model accounts for finite heat transfer rates, heat leaks, and friction as different sources of dissipation. The performance characteristics are cast in terms of cooling rate $(r)$ versus coefficient of performance $(w)$. For comparison purposes, various types of refrigeration/heat pump systems are considered: the thermoelectric refrigerator, the reverse Brayton cycle, and the reverse Rankine cycle. Although the dissipation mechanisms are different (e.g., heat leak and Joule heating in the thermoelectric refrigerator, isentropic losses in the reverse Brayton cycle, and limits arising from the equation of state in the reverse Rankine cycle), the $r-w$ characteristic curves have a general loop shape. There are four limiting types of operation: open circuit in which both $r$ and $w$ vanish in the limit of slow operation; short circuit in which again $r$ and $w$ vanish but in the limit of fast operation; maximum $r$; maximum $w$. The behavior of the considered systems is explained by means of the proposed model. The derived formulae could be used for a quick estimation of $w$ and the temperatures of the working fluid at the hot and cold sides.
\end{abstract}

\section{Introduction}

Classical thermodynamics places bounds on thermodynamic measures based on reversible assumptions $[1,2]$. The resulting bounds often have limited practical value because reversible operation means either zero rate of operation or infinite system size. Finite-time thermodynamics (FTT) extends thermodynamic analysis to include finite-time constraints, for example, finite heat transfer rates, heat leaks, and friction while maintaining a finite process/cycle time [3]. These methods derive more realistic upper bounds on performance, often including the path for achieving such upper bounds. The methods of FTT have been applied to numerous thermodynamic systems [4-19]. Heat engines were treated extensively, and an expression for the efficiency at maximum power production of a heat engine that operates between two heat reservoirs at temperatures $T_{H}$ and $T_{C}$ under the limitation of Newtonian heat transfer was found to be given by the simple expression [4]

$$
\eta_{C A}=1-\sqrt{\frac{T_{C}}{T_{H}}} .
$$

Also refrigeration/heat pump systems were originally treated with finite heat transfer rate as the sole dissipation mechanism [15-18]. Later, reciprocating chillers [19] were analyzed and their characteristics compared to experimental data. The effect of heat leak and friction on the performance of heat engines were addressed by $[5,11,12]$. The effect of heat leak on the performance of refrigeration/heat pump systems was also addressed by [14], and their characteristic flows were described using power degradation or power coefficient of performance coordinates.

A number of studies addressed analyses of different refrigeration systems using finite-time methods under different modes of irreversibility and for different heat transfer laws. These studies include optimization of endoreversible and irreversible vapor absorption refrigeration systems with external and internal irreversibility [20] and an exergetic efficiency optimization of an irreversible heat pump working on a reversed Brayton cycle [21]. Their general performance characteristics were reported in [22]. Optimal performance of a Carnot heat pump under the condition of mixed heat resistance [23], a nonendoreversible Carnot refrigerator at 


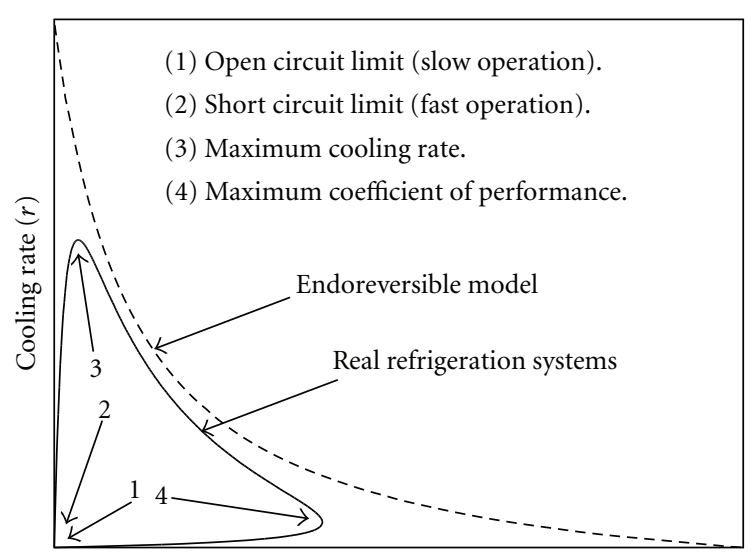

Coefficient of performance $(w)$

Figure 1: Cooling rate versus coefficient of performance for the endoreversible heat pump model where finite heat transfer rate is the sole source of losses (dashed curve) and for real refrigeration systems (solid curve). There are four distinct limiting operation points for real systems: The open circuit limit (heat leak dominated); maximum $w$ (coefficient of performance); maximum $r$ (cooling rate); the short circuit limit (friction dominated).

maximum cooling power [24], and a fundamental optimal relation of a generalized irreversible Carnot heat pump with complex heat transfer law [25] are further examples based on this ideal cycle. These were contrasted in [26] by an optimization of real operating refrigeration machines.

Several other objective functions have been proposed. An "ecological" function combines maximization of power with minimization of losses (entropy production) [27]. A related "ecological coefficient of performance," defined as the ratio of the cooling load to the rate of availability loss (or entropy generation rate) [28], has considerable intuitive merit. Compactness is favored by the objective function "power density", that is, power produced relative to maximum volume of the cycle [29]. Economic considerations were introduced by a thermoeconomic optimization of a two-stage combined refrigeration system [30] and by a profit rate performance optimization of a generalized irreversible refrigeration/heat pump cycle [31].

In the present study, the finite-time analysis of refrigeration/heat pump systems is extended to include friction, solid, and fluid, as another important source of dissipation. The characteristics are demonstrated in cooling rate $(r)$ versus coefficient of performance $(w)$ coordinates. The observed characteristics are compared to the characteristics of real refrigeration/heat pump devices, and simple formulae for quick estimate of $w$ and the temperatures of the working fluid at the hot and cold sides are proposed.

We show that refrigeration/heat pump devices have a common generalized performance characteristic, as derived from the aforementioned generic model in finite time. For comparison purposes, different thermodynamic systems of refrigeration/heat pump are analyzed, for example, the thermoelectric refrigerator, the reversed Brayton cycle, and the reversed Rankine cycle. In each cycle, the major dissipation mechanisms are considered, for example, heat leak and
Joule heating in the thermoelectric refrigerator, isentropic losses or inefficiency due to fluid friction in the turbine and compressor in the reversed Brayton cycle, and the limitations that arise from the equation of state in the reversed Rankine cycle. For each cycle, the $r-w$ curve is derived using FTT. The general shape of this curve is shown in Figure 1 for the endoreversible generic model in which finite heat transfer rate is the sole source of dissipation (dashed curve), along with the loop-type shape for real systems (solid curve).

Apparently, there are four limiting types of operation: open circuit in which both $r$ and $w$ vanish in the limit of slow operation; short circuit in which again $r$ and $w$ vanish but in the limit of fast operation; maximum $r$; maximum $w$. For some real devices, it is not possible to measure all four operating points due to practical limitations, for example, a maximum temperature limit or a maximum pressure limit.

The current analysis falls into two major parts. First, to gain theoretical and basic understanding of refrigeration/heat pump systems, we analyze different types of systems and highlight the major dissipation mechanisms for the different cycles. By a sensitivity study of the effect of the losses, the potential improvements are demonstrated. Second, a generic model is proposed to describe the behavior of practical refrigeration/heat pump systems. The derived formulae can be used for quick estimates of the performance criteria of real systems, primarily in the early stages of design.

The paper is arranged as follows. In Section 2, the methodology and basic definitions are introduced. In Section 3, the thermoelectric refrigerator is considered. In Section 4, we analyze the reversed Brayton cycle. In Section 5, the reversed Rankine cycle is discussed. In Section 6, the generic model in finite time to describe the characteristics of real refrigeration/heat pump systems including heat leak and friction is studied, and finally conclusions are given in Section 7.

\section{Methodology}

A refrigeration/heat pump system is a device that removes heat from a cold reservoir and delivers heat to a hot reservoir. This is accomplished by applying power input to the machine. An elementary picture of a heat pump is made up of a heat exchanger to a cold reservoir at temperature $T_{C}$, a heat exchanger to a hot reservoir at temperature $T_{H}$, a "compressor," and an "expander". The cooling rate $r$ of a refrigeration system is defined as the instantaneous cooling power or the average amount of heat that is extracted from the cold reservoir per cycle time. The efficiency of the refrigeration systems is measured in terms of the coefficient of performance $w$ defined as the ratio between the cooling rate and the power input $P$ to the machine.

In this work, different cooling devices are analyzed with their major dissipation mechanisms. For each cycle type, the $r-w$ characteristic curve is derived based on FTT. The power input to the machine is derived from the energy balance (first law of thermodynamics) for a cyclic process and is given by

$$
P=h-r
$$


where $h$ is defined as the rate of heat discharged to the hot reservoir. The second law of thermodynamics for a cyclic process requires the entropy balance

$$
\int_{0}^{\tau}\left(\frac{h}{T_{h}}-\frac{r}{T_{c}}\right) d t=0
$$

where $\tau$ is the cycle time, $T_{h}$ is the temperature of the working fluid on the hot branch, and $T_{c}$ is the temperature of the working fluid on the cold branch of the cycle. Finally, the coefficient of performance is defined as

$$
w=\frac{r}{P} .
$$

As stated earlier, the $r-w$ characteristic curves will be derived for the different refrigeration/heat pump systems, and their general behavior is discussed in the following sections.

\section{The Thermoelectric Refrigerator}

In 1822, Seebeck observed that if a closed circuit is made of two dissimilar metals, an electrical current will flow in the circuit when the two junctions are maintained at different temperatures [32]. In 1834, Peltier observed the inverse effect. If an electrical current flows across the isothermal junction between two dissimilar metals, heat is either absorbed or produced [32]. In 1851, Thomson pointed out a third effect which relates heat absorbed or produced in a conductor to the temperature gradient along it and the current flowing through it [32]. The Thomson effect is small compared to the Peltier effect in thermoelectric cooling and will be neglected here.

Seebeck Effect. For a small temperature difference between the two junctions of any materials $A$ and $B$, the open circuit voltage $V$ developed is proportional to the temperature difference $\Delta T$ and is given by

$$
V=\alpha_{A B} \Delta T,
$$

where $\alpha_{A B}$ is the difference between the absolute Seebeck coefficients for materials $A$ and $B$ (semiconductors are usually used for thermoelectric systems).

Peltier Effect. In the reverse process where the junction is connected to a battery to provide a direct current $I$, heat will be absorbed or produced at the junctions between the dissimilar materials. The absorbed or produced heat is proportional to the electrical current. Lord Kelvin showed that the proportionality constant is given by the product of the Seebeck coefficient and the absolute temperature $T$. Hence, heat $Q$ absorbed or produced per unit time at the junctions between two dissimilar materials is given by

$$
Q=\alpha_{A B} I T .
$$

There are two additional effects always present in a real thermoelectric circuit which limit its performance: the Joule heating effect (ohmic resistance) and heat leak between the

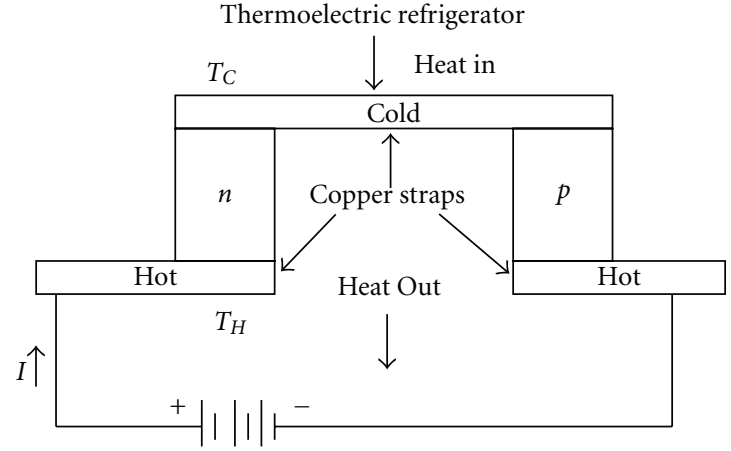

FIgURE 2: Schematic of the thermoelectric refrigerator.

two junctions. The best materials for thermoelectric devices are semiconductors which provide a compromise between the two conflicting requirements, a good electric conductor and a good thermal insulator.

A complete circuit (Figure 2) is constructed from two dissimilar materials, for example, $n$ and $p$ type semiconductors. There are two junctions in the circuit, a hot and a cold junction where heat is produced and absorbed, respectively. The temperature gradient between the hot and cold junctions is not linear because of production of Joule heat within each conductor.

By considering the steady state condition at the junctions and the total input power, the characteristics of such a couple may be deduced. The following assumptions are made: the resistivity and thermal conductivity of the materials are independent of temperature, and heat exchange between the thermocouple and its environment occurs only at the hot and cold junctions.

Steady state balance at the cold junction, which is the Peltier cooling, is made up of the heat conducted through the couple conductors plus the ambient heat absorbed (useful heat pumped):

$$
\alpha T_{C} I=\kappa \Delta T+\frac{I^{2} R}{2}+r
$$

or

$$
r=\alpha T_{C} I-\kappa \Delta T-\frac{I^{2} R}{2},
$$

where $\kappa$ is the thermal conductance of thermocouple conductors, $I$ is the electrical current, $r$ is the rate of heat absorbed at the cold junction, $R$ is the electrical resistance of couple conductors, $T_{C}$ is the cold junction temperature, $T_{H}$ is the hot junction temperature, $\Delta T=T_{H}-T_{C}$ is the operating temperature difference, and $\alpha=\alpha_{p n}=\alpha_{p}-\alpha_{n}$ is the difference between the absolute Seebeck coefficients of the $p$ and $n$ materials.

The power $P$ required to obtain the rate of cooling of (8) is given by

$$
P=I V=I(I R+\alpha \Delta T),
$$

where $V$ is the voltage applied across the junctions. The applied voltage is the sum of two terms, the voltage drop 
which always occurs in an electrical conductor, plus that required to overcome the Seebeck voltage. The coefficient of performance $w$ is thus given by (4),

$$
w=\frac{r}{P} \text {. }
$$

The so-called figure of merit $Z$ of the thermocouple

$$
Z=\frac{\alpha^{2}}{\kappa R}
$$

is the most important overall parameter associated with a thermoelectric material [32]. The higher it is the better the performance of the thermoelectric couple. It is obvious from (11) that a high Seebeck coefficient, low electrical resistivity, and low thermal conductivity are essential.

For convenience, we define the following dimensionless quantities:

$$
\begin{gathered}
r^{*}=\frac{r R}{\alpha^{2} T_{C}^{2}}, \\
I^{*}=\frac{I R}{\alpha T_{C}}, \\
T_{C}^{*}=\frac{T_{C}}{T_{H}}, \\
P^{*}=\frac{P R}{\alpha^{2} T_{C}^{2}}, \\
Z^{*}=\frac{Z\left(T_{C}+T_{H}\right)}{2} .
\end{gathered}
$$

Using the definitions equations (12), (8)-(10) become

$$
\begin{gathered}
r^{*}=I^{*}-I^{* 2} / 2-\frac{\left(1 / T_{C}^{* 2}-1\right)}{2 Z^{*}}, \\
P^{*}=I^{*}\left(\frac{1}{T_{C}^{*}}-1+I^{*}\right), \\
w=\frac{I^{*}-I^{* 2} / 2-\left(1 / T_{C}^{* 2}-1\right) / 2 Z^{*}}{I^{*}\left(1 / T_{C}^{*}-1+I^{*}\right)} .
\end{gathered}
$$

Figure 3 presents sample plots of $r^{*}$ versus $w$ for different values of the figure of merit $Z^{*}$. Typical values of $Z^{*}$ for practical materials are close to 1.0. In this paper, different values of $Z^{*}$ in the range 0.3 to $\infty\left(Z^{*}=\infty\right.$ corresponds to no heat leak losses) are considered, and the potential improvements are shown in Figure 3.

From Figure 3, we recognize the four major operation points (as marked in Figure 2): open circuit limit for infinitely slow operation or zero electric current in which case both $r^{*}$ and $w$ vanish while heat leak dominates; short circuit limit for fast operation where again $r^{*}$ and $w$ vanish and frictional effects dominate; maximum $w$; maximum $r^{*}$ operating points.

It is important to note that for practical values of $Z^{*}$ the value of the maximum coefficient of performance is not very different from the value of the coefficient of performance at maximum cooling rate but that the two values separate as the value of $Z^{*}$ becomes larger. The maximum $w$ point reaches the reversible limit when $Z^{*}$ becomes infinite; in this limit, the effect of heat leak is negligible.

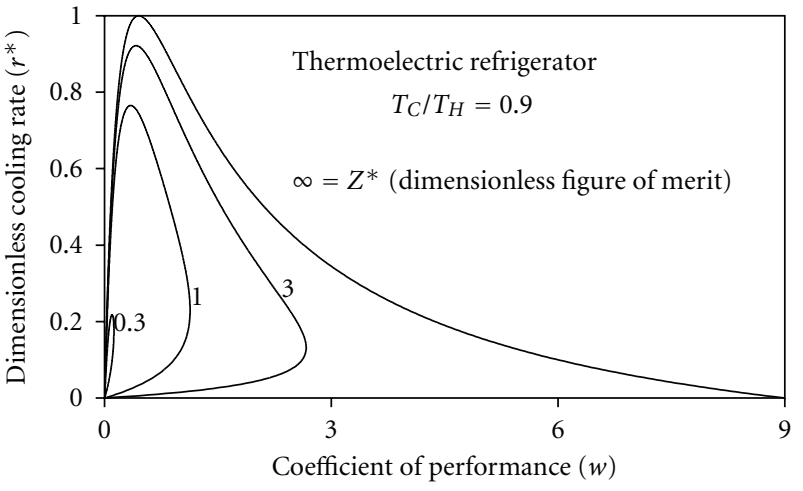

FIGURE 3: Dimensionless cooling rate $\left(r^{*}\right)$ versus coefficient of performance $(w)$ for the thermoelectric refrigerator. Plots are presented for different values of the figure of merit $\left(Z^{*}\right)$ in the range $0.3-\infty$, where $Z^{*}=\infty$ corresponds to lossless operation.

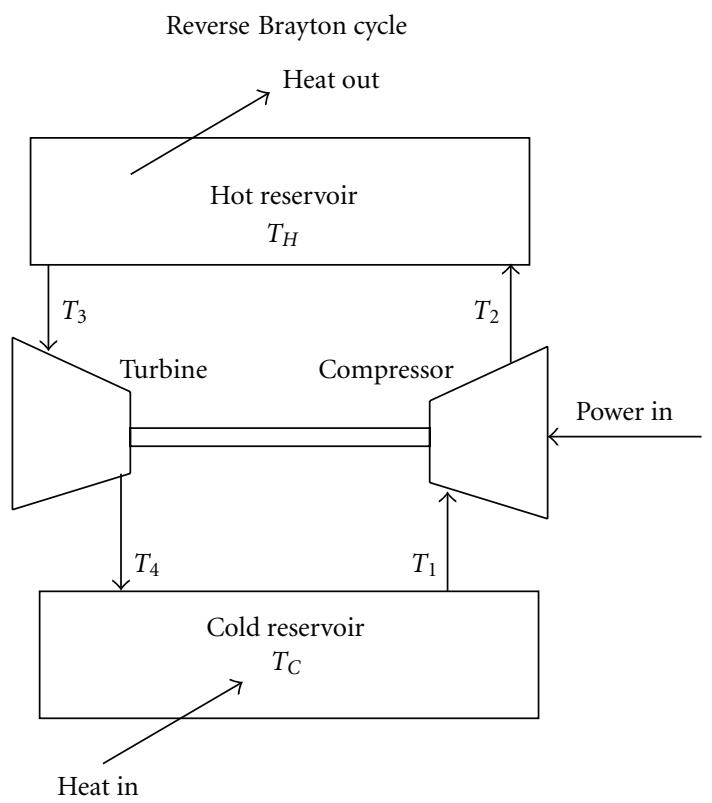

FIGURE 4: Schematic of the reverse open Brayton cycle.

\section{The Reverse Brayton Cycle}

An open reverse Brayton cycle is considered with air as the working fluid. For simplicity, we assume that the working fluid is an ideal gas. Figure 4 is a schematic diagram of the cycle and shows its basic elements.

The basic cycle assumes reversible operation of the compressor and turbine. These ideal processes are assumed to be isentropic, that is, reversible adiabatic processes. The observed $r-w$ curve following these assumptions is similar to the observed curve from the endoreversible model for refrigeration systems.

In estimating the performance of real cycles, pressure losses should be accounted for in heat exchangers, regenerators, and in ducting. In this paper, such losses will not be included but are assumed to be minimal [33]. The major losses considered here are due to imperfections of the turbo 
machinery, the compressor, and the turbine. The losses are conventionally measured in terms of the isentropic efficiency (which indicates the deviations from isentropic processes due to fluid friction) of the turbine and compressor. The thermodynamic analysis of the basic heat pump model may be summarized as follows.

The equation of state for an ideal gas with the assumption of isentropic processes (reversible adiabatic processes or, equivalently, constant entropy processes) leads to

$$
p V^{\gamma}=\text { const, }
$$

where $p$ is the pressure, and $V$ is the volume of the working fluid, respectively. $\gamma$ is the ratio between the specific heat at constant pressure and the specific heat at constant volume of the working fluid. The isentropic temperature ratio $a$ is defined as

$$
\alpha \equiv \frac{T_{2}}{T_{1}} \equiv \frac{T_{3}}{T_{4}} \equiv\left(\frac{p_{3}}{p_{4}}\right)^{(\gamma-1) / \gamma} \equiv\left(\frac{p_{2}}{p_{1}}\right)^{(\gamma-1) / \gamma},
$$

where $T_{i}, i=1, \ldots, 4$ are the temperatures of the working fluid at different stages of the cycle, as shown in Figure 4.

Applying the first law of thermodynamic to the turbine and compressor processes, the average net power is derived and is given by [33]

$$
P=\dot{m} C_{p} T_{3}\left(\frac{T_{C}^{*}}{\eta_{c}}-\frac{\eta_{t}}{a}\right)(a-1),
$$

where $\dot{m}$ is the flow rate, and $C_{p}$ is the specific heat at constant pressure condition of the working fluid, respectively; $T_{C}^{*}$ is the ratio between $T_{3}$ and $T_{1} ; \eta_{t}$ and $\eta_{c}$ are the isentropic efficiencies of the turbine and compressor, respectively.

First law analysis of the cold reservoir leads to the expression for the average cooling rate

$$
r=\dot{m} C_{p} T_{3}\left(T_{C}^{*}-1+\eta_{t}\left(1-\frac{1}{a}\right)\right) .
$$

Finally, the coefficient of performance is given by

$$
w=\frac{r}{P}=\frac{T_{C}^{*}-1+\eta_{t}(1-1 / a)}{(a-1)\left(T_{C}^{*} / \eta_{c}-\eta_{t} / a\right)} .
$$

Examination of the expressions for the cooling rate and coefficient of performance shows that $w$ vanishes at both limits of slow and fast operation, whereas $r$ vanishes only in the limit of slow operation, the open circuit limit. For slow operation, the isentropic temperature ratio $a$ is slightly above unity as derived from the requirement of positive cooling rate. The fast speed limit is achieved by an infinite value of $a$ for which $w$ vanishes but not $r$. The resulting characteristics are shown by the $r-w$ curves in Figure 5 . The cooling rate (relative to its maximum value in the case of ideal processes, i.e., $\eta_{t}=1$ ) is plotted versus the coefficient of performance for different values of isentropic efficiency.

For simplicity, the isentropic efficiencies of the turbine and the compressor are chosen equal. The range of these efficiencies is chosen in the range $0.8-1.0$ to represent

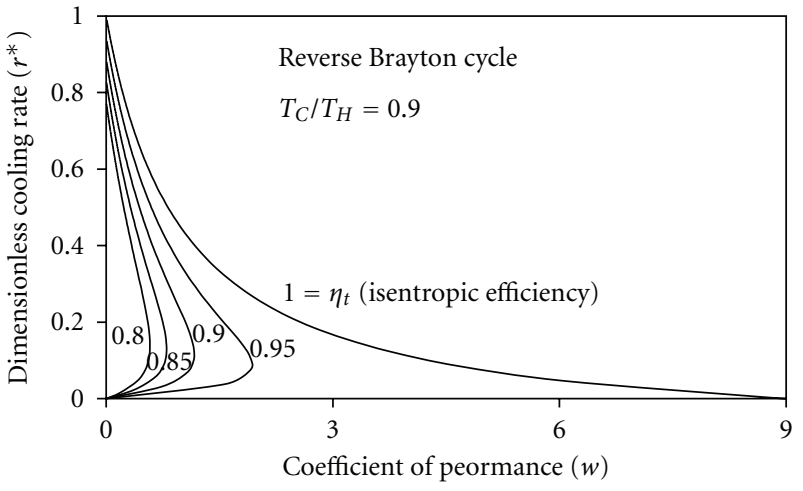

FIgURE 5: Dimensionless cooling rate $\left(r^{*}\right)$ versus coefficient of performance $(w)$ for the reverse Brayton cycle. Plots are presented for different values of the isentropic efficiency $\eta_{t}$ in the range $0.8-$ 1.0 , where $\eta_{t}=1$ corresponds to reversible operation.

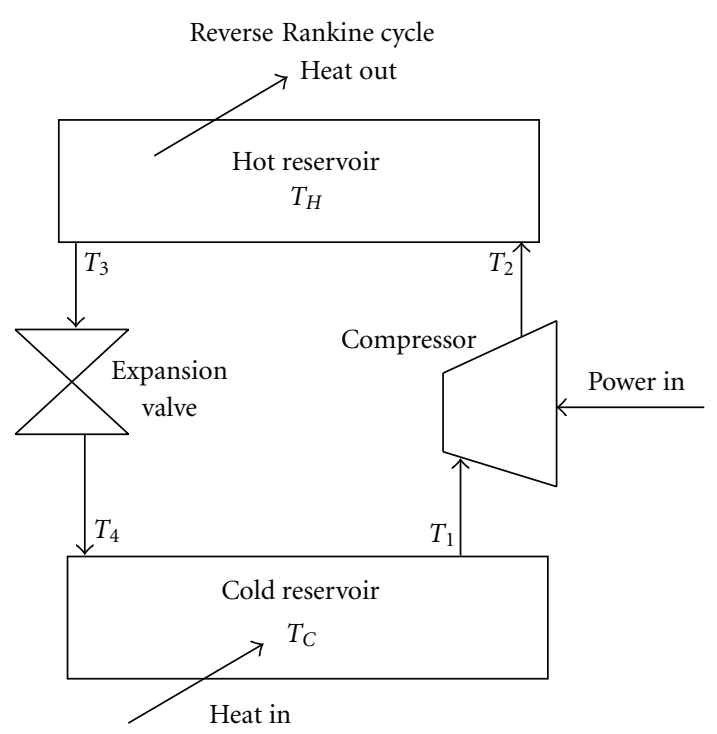

Figure 6: Schematic of the reverse Rankine cycle.

practical values, approaching the ideal limit of 1 . Although the full characteristic curve is shown, it is possible to measure only part of it due to practical limitations, that is, maximum pressure and maximum temperature limits. The observed maximum point of the coefficient of performance is small (compared to the maximum point of reversible processes) for practical values of isentropic efficiency $(0.8-0.9)$. The potential improvements are evident in Figure 5.

\section{The Reverse Rankine Cycle}

The reverse Rankine cycle is widely used for heating and cooling in heat pump, air conditioning, and refrigeration systems. The basic elements of the cycle are a compressor, a condenser (hot reservoir), an expansion valve, and an evaporator (cold reservoir) (see Figure 6). The thermodynamic analysis of the Rankine cycle may be based on three different principles. (i) The Carnot cycle which assumes isothermal 


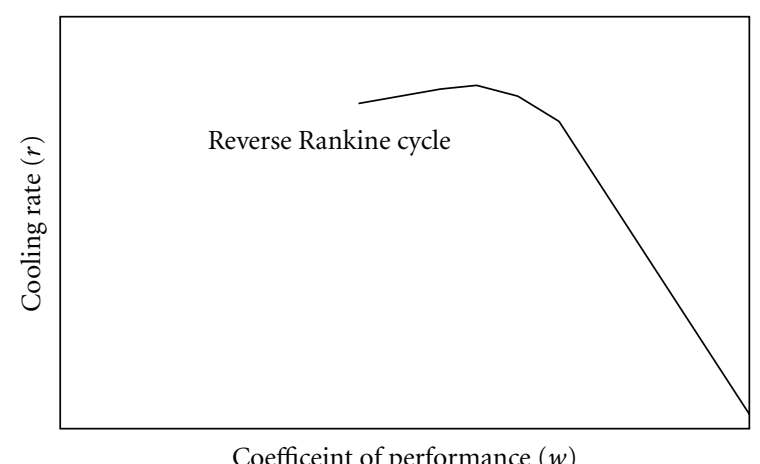

Figure 7: Dimensionless cooling rate $\left(r^{*}\right)$ versus coefficient of performance $(w)$ for the reverse Rankine cycle. The losses arise due to the real equation of state.

heat addition and rejection processes, coupled with reversible adiabatic expansion and compression, respectively. The maximum coefficient of performance depends on the ratio of the reservoir temperatures

$$
w_{\text {rev }}=\frac{T_{C} / T_{H}}{1-T_{C} / T_{H}},
$$

where $T_{C}$ and $T_{H}$ are the cold reservoir temperature and the hot reservoir temperature, respectively. This result is useful primarily as the ultimate reference with which to compare the performance of real cycles. (ii) The other extreme would be an experimental comparison of refrigerants in full-size equipment. While this approach would be necessary to fully evaluate a fluid, it is not suited for an initial screening of fluids because of the large number of equipment parameters, in addition to fluid properties, which would influence the performance. (iii) An intermediate approach is required. The next level of detail beyond the Carnot cycle is the theoretical vapor compression cycle. This assumes a reversible isentropic compression process while the condenser and evaporator operate at constant pressure with the refrigerant exiting as saturated liquid and vapor, respectively. The reversible expansion process of the Carnot cycle, typically carried out by a turbine, is here replaced by an irreversible expansion at constant enthalpy, representing a simple throttle. The theoretical vapor compression cycle can be extended to include heat transfer limitations. A practical vapor compression cycle would account for the effects of pressure drop, compressor isentropic efficiency, condenser subcooling, and evaporator superheating. This approach could permit a comparison of refrigerants taking into account most of the nonidealities present in real systems, but with the equipment specified in a generic fashion. Working with reduced quantities [34] for a characteristic refrigerant, all possible working fluids may be considered at the same time. The principle of corresponding states provides a way to compare various fluids on a fundamental basis. This principle expresses the observation that, when scaled in terms of reduced properties, many fluids are nearly identical. (A reduced property is the ratio of a quantity to its value at the critical point.)
A model of the reverse Rankine cycle has been analyzed for a variety of different working fluids based on the assumption of corresponding states [34]. The real equation of state of the refrigerant working fluid was assumed to be of the Carnahan-Starling-Desantis (CSD) form. This equation of state is given as (1) and coupled with (2)(5) and Figures $1-3$ of [34]. For a given ratio $T_{C} / T_{H}$, the performance characteristics, that is, heating load and coefficient of performance, were calculated as functions of the condenser temperature. The results were presented using the heating load and the coefficient of performance for heating versus the reduced condenser temperature (Figures 5 and 6 in [34]). The cooling rate versus coefficient of performance is extracted from those plots for the case of $T_{C} / T_{H}=0.9$ and recast using $r-w$ coordinates (Figure 7).

The conclusion from this analysis was that the maximum $w$ operation point is not observed, but there is a maximal cooling rate. Besides the limitation of the equation of state, the Rankine cycle suffers mainly from losses in the expansion valve which is an irreversible adiabatic process (constant enthalpy process). This could be viewed as a friction type dissipation source which leads to a maximum in cooling rate (fast operation mode). This role of friction is exhibited also by the thermoelectric refrigerator.

A key feature of the Rankine cycle is a phase change in the working fluid [34], and the two isothermal steps correspond to condensation of the fluid at $T_{H}$ and evaporation at $T_{C}$. The cycle has intrinsic irreversibilities associated with the free expansion of the liquid to the temperature at which condensation occurs. The largest reduction in $w$ occurs in the heat exchangers due to finite temperature differences.

In the next section, a generic model for refrigeration/heat pump devices will combine all of the previously considered effects into one simple model.

\section{Finite-Time Model for Refrigeration/Heat Pump Systems}

In this section, we present a finite-time model to describe the principal characteristics of real refrigeration/heat pump systems. The irreversibilities are due to finite heat transfer rates, heat leak, and friction, fluid as well as solid.

Consider a generic heat pump which operates between two heat reservoirs, the hot reservoir at temperature $T_{H}$ and the cold reservoir at $T_{C}$. The temperature of the working fluid on the hot side $T_{h}$ is higher than the reservoir's temperature due to finite rate of heat transfer. The temperature of the working fluid on the cold side $T_{c}$ is lower than the temperature of the cold reservoir, again due to finite heat transfer rates. In addition, there is a heat leak directly from the hot reservoir to the cold reservoir. As an example to understand this term, consider the household refrigerator. Heat leaks constantly from the surroundings (hot reservoir) into the inside of the refrigerator (cold reservoir). When the compressor operates, this heat leak is negligible compared to the refrigeration. However, during the period when the compressor is not operating, the heat leak is the only process so its effect is no longer negligible but rather determines 
the time period between compressor operations. The third dissipation term considered in the present model is due to frictional losses which would arise from rubbing friction of the moving parts or from fluid friction of the working fluid. In the present study, the frictional dissipation term is taken proportional to the square of the power input to the machine. The effect of friction becomes important at high speeds.

The heat rejected to the heat reservoirs has three contributions: finite heat transfer rate, heat leak, and friction. On the hot side, friction gives a positive contribution which could be explained as follows: the heat generation due to friction is pumped to the hot reservoir, and the heating load is higher than that required from reversible operation. On the other hand, the cooling rate is reduced due to friction. The balance equations according to the first and second laws of thermodynamics are summarized in the following equations.

The rate of heat rejection

$$
h=\kappa_{H}\left(T_{h}-T_{H}\right)+\phi_{H} P^{2}-\kappa_{L}\left(T_{H}-T_{C}\right),
$$

where $\kappa_{H}$ and $\phi_{H}$ are the heat conductance between the hot reservoir and the working fluid and the frictional coefficient at the hot side of the system, respectively, while $\kappa_{L}$ is the heat leak conductance between the hot and cold reservoirs.

The cooling rate

$$
r=\kappa_{C}\left(T_{C}-T_{c}\right)-\phi_{C} P^{2}-\kappa_{L}\left(T_{H}-T_{C}\right)
$$

where $\phi_{C}$ is the friction coefficient at the cold side of the system.

Finally, the power input

$$
P=h-r=\kappa_{H}\left(T_{h}-T_{H}\right)-\kappa_{C}\left(T_{C}-T_{c}\right)+\left(\phi_{H}+\phi_{C}\right) P^{2} .
$$

The second law now gives for a full cycle $\Delta S=0$ or

$$
\frac{k_{H}\left(T_{h}-T_{H}\right)}{T_{h}}=\frac{k_{C}\left(T_{C}-T_{c}\right)}{T_{c}},
$$

Using the following definitions

$$
\begin{gathered}
T^{*}=\frac{T}{T_{H}}, \quad P^{*}=\frac{P}{\left(\kappa_{H} T_{H}\right)}, \\
x^{*}=T_{h}^{*}-1, \quad y^{*}=T_{C}^{*}-T_{c}^{*}, \\
\eta_{C}=1-T_{C}^{*}, \quad \eta_{0}=1-\frac{y^{*}}{x^{*}}, \\
\kappa_{L}^{*}=\frac{\kappa_{L}}{\kappa_{H}}, \quad \kappa^{*}=\frac{\kappa_{H}}{\kappa_{C}}, \\
r^{*}=\frac{r}{\left(\kappa_{H} T_{H}\right)}, \quad h^{*}=\frac{h}{\left(\kappa_{H} T_{H}\right)}, \\
\phi_{C}^{*}=\phi_{C} \kappa_{H} T_{H}, \quad \phi_{t}^{*}=\left(\phi_{H}+\phi_{C}\right) \kappa_{H} T_{H} .
\end{gathered}
$$

Equations (22)-(24) can be rearranged to the following form:

$$
\begin{gathered}
x^{*}=\frac{\eta_{0}-\eta_{C}}{\left(1+1 / \kappa^{*}\right)\left(1-\eta_{0}\right)}, \\
y^{*}=\frac{\eta_{0}-\eta_{C}}{\left(1+\kappa^{*}\right)}, \\
P^{*}=\frac{\left(1 \pm \sqrt{1-4 \eta_{0}\left(\eta_{0}-\eta_{C}\right) \phi_{t}^{*} /\left(1+1 / \kappa^{*}\right)\left(1-\eta_{0}\right)}\right)}{2 \phi_{t}^{*}}, \\
r^{*}=\frac{\eta_{0}-\eta_{C}}{1+1 / \kappa^{*}}-\kappa_{L}^{*} \eta_{C}-\phi_{C}^{*} P^{* 2} .
\end{gathered}
$$

Finally, the coefficient of performance is given by

$$
w=\frac{r^{*}}{P^{*}}=\frac{r}{P} .
$$

\section{Heat Leak Effect Only}

For the case of no frictional dissipation terms, $\phi_{t}^{*}=\phi_{C}^{*}=0$, the expression of the coefficient of performance simplifies to

$$
w=\left(\frac{1}{\eta_{0}}-1\right)\left(1-\frac{D}{\eta_{0}-\eta_{C}}\right),
$$

where $D \equiv \eta_{C} \kappa_{L}^{*}\left(1+1 / \kappa^{*}\right)$ and the cooling rate is

$$
r^{*}=\frac{\eta_{0}-\eta_{C}-D}{1+1 / \kappa^{*}} .
$$

The coefficient of performance has a maximum at the point where

$$
\eta_{0}=\frac{\eta_{c}+D}{1+D}\left(1+\sqrt{1-\frac{\eta_{c}(1+D)}{\eta_{c}+D}}\right) .
$$

This clearly approaches the Carnot efficiency $\eta_{C}$ as the leak conductance $\kappa_{L}$, and thus $D$, vanishes.

Equations (26), (27), and (31) coupled with (24) could be used for a quick estimate of the temperature of the working fluid on the hot branch, the temperature of the working fluid on the cold branch, and the coefficient of performance, respectively, based on two parameters (assuming $\kappa^{*}=1$ ): the reservoir temperature ratio $T_{C}^{*}$ and the relative value of heat leak $\kappa_{L}^{*}$.

To demonstrate the usage of these equations, we consider the following numerical example. For a real refrigeration system, a typical value of the ratio of heat reservoir temperatures $T_{C}^{*}=0.9$, the relative value of heat leak to the conductance of the hot side is assumed to be small, and we consider the value of $\kappa_{L}^{*}=0.1$, along with equal heat conductances on the hot and cold sides $\left(\kappa^{*}=1\right)$. A typical ambient temperature is $T_{H}=35^{\circ} \mathrm{C}$. Based on these values the temperature of the cold space is $T_{C}=4.2^{\circ} \mathrm{C}$, and the resulting working fluid temperatures are $T_{h}=46.6^{\circ} \mathrm{C}, T_{c}=-5.5^{\circ} \mathrm{C}$. Finally, the estimated $w$ is 3.5 which is about 2.5 times less than the value of the reversible limit of 9 in this example. 


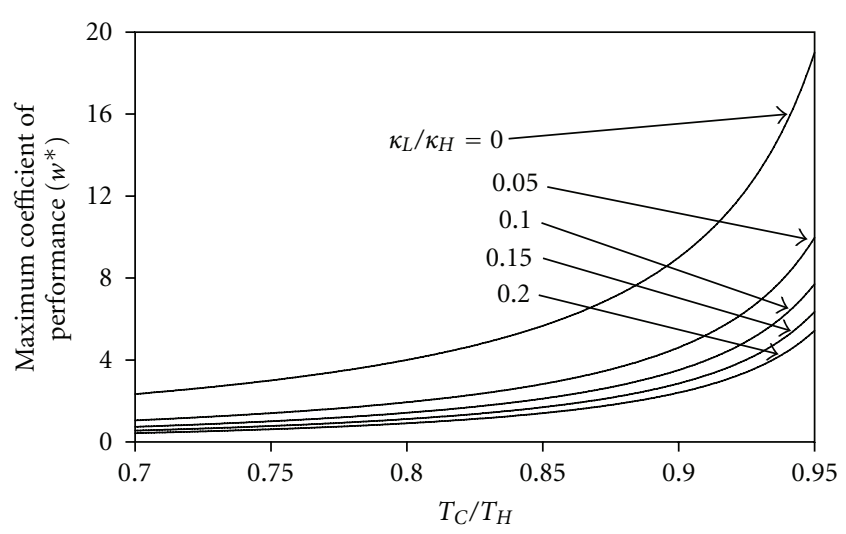

Figure 8: Maximum coefficient of performance $w$ versus the ratio of the reservoir temperatures $\left(T_{C}^{*}=T_{C} / T_{H}\right)$ as derived from the FTT model. Plots are presented for different values of the heat leak.

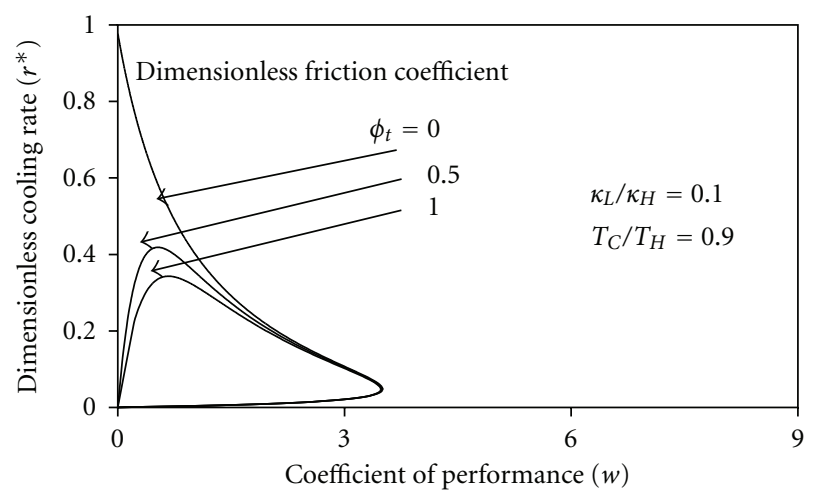

FIGURE 9: Dimensionless cooling rate $\left(r^{*}\right)$ versus coefficient of performance $(w)$ for the generic model designed to describe the behavior of real refrigeration systems in finite time. The assumed sources of loss are: Finite heat transfer rates, heat leak, and friction. Plots are presented for different values of friction while heat leak is kept constant at $\kappa_{L}^{*}=0.1$.

This example illustrates that even small values of the heat leak coefficient induce a large reduction in $w$. The assumed value of $\kappa_{L}^{*}=0.1$ is based on the assumption of a desired heat leak ratio of $10^{-4}$ and a time duration between compressor operations of 1200 seconds.

The maximum coefficient of performance is plotted versus $T_{C}^{*}$ in Figure 8 . For the case of zero heat leak, the reversible limit is recovered.

\section{Effect of Friction}

In addition to finite heat transfer rates and heat leak, friction is another important source of dissipation. We assumed that the rate of friction dissipation is proportional to the square of the required power input. Using (26)-(28), the $r-w$ curve is plotted for the case of heat leak $\kappa_{L}^{*}=0.1, \phi_{c}=\phi_{t} / 2$, and the results are presented by Figure 9. The $r-w$ curves are plotted for different strengths of friction. The frictional losses as modeled here dominate at fast operation where both the cooling rate and the coefficient of performance vanish. It is clear from Figure 9 that the effect of friction at the maximum $w$ point is negligible. Figure 9 is a typical plot of cooling rate (relative to its maximum value for zero heat leak) versus coefficient of performance for different strengths of friction. For slow operation, the heat leak is dominating, and both the cooling rate and the coefficient of performance vanish. In the other limit of fast operation, again both the cooling rate and the coefficient of performance vanish due to friction (friction-dominated regime). Although the full cycle is plotted here, it is important to remember the limitations on temperature which lead to termination of the calculation at some point on the plot. Obviously, no temperatures can be negative, and materials put upper bounds on realistic temperatures as well.

The generic model in finite time explains the behavior of the $r-w$ curve of real refrigeration/heat pump systems based on the assumption of three main dissipation terms: finite heat transfer rates, heat leaks, and friction. It is observed that without friction the fast operation limit does not correspond to realistic performance. Real refrigeration/heat pump systems work in the slow operation regime due to practical limitations of material properties.

\section{Conclusions}

In this paper, we analyze a series of idealized refrigeration/heat pump systems. Different thermodynamic cycles and models are considered to include various dissipation mechanisms. Although it is not possible to measure the full characteristic curve (as described here using $r-w$ coordinates) for all real refrigeration/heat pump systems, they all have a general type of behavior (Figure 1, solid line). The limitations are due to a maximum temperature (thermal) and a maximum pressure (mechanical).

For the considered refrigeration/heat pump systems, we observe four special operation points (as pointed out in Figure 1): slow operation (heat leak-dominated regime) in which both the cooling rate and the coefficient of performance vanish; fast operation (friction-dominated regime) in which again the cooling rate and the coefficient of performance vanish; in between these two extreme points exist the maximum coefficient of performance and the maximum cooling rate points, respectively.

Due to practical limitations, most of the cooling systems are designed to operate in the slow regime. The aforementioned points are illustrated using different types of systems.

The thermoelectric refrigerator is a good example of a real heat pump, where it is possible to measure the four operation points (Figure 3). The major dissipation mechanisms in the thermoelectric refrigerator are heat leak and Joule heating (ohmic resistance/friction-like term). The most important parameter for thermoelectric refrigerators is the figure of merit $Z$. High $Z$ values could be achieved with higher values of the Seebeck coefficient, low thermal conductivity (heat leaks), and low electric resistance. Typical values of the dimensionless figure of merit (12) for thermoelectric devices are in the range $0.3-1.0$. 
The reverse Brayton cycle, which could be visualized as a finite heat reservoir, mainly suffers from imperfections of the compressor and the turbine (fluid friction). These imperfections are described using the isentropic efficiency of the turbine and compressor, their typical values being in the range $0.8-0.9$. For this cycle in the slow operation mode, both $w$ and $r$ vanish while at the fast operation limit only $w$ vanishes (Figure 5).

The limitation in the Rankine cycle arises from the real equation of state of the working fluid. Besides this limitation, there are other losses basically due to finite heat transfer rates and expansion losses. For the Rankine cycle analysis, assuming a generalized equation of state (based on the assumption of corresponding states), only the maximum cooling rate point was observed (Figure 7).

Finally, a new general finite-time model is analyzed. The dissipation mechanisms are finite heat transfer rates, heat leak, and friction. As a generalized model, it exhibits the four operating points of real refrigeration/heat pump systems. The effect of finite heat transfer rates is considered first, and the derived $r-w$ curve is presented for different values of heat leak (Figure 8). Within these assumptions, the proposed model displays two limit points only, the slow operation limit and maximum $w$ point. The derived expressions for the working fluid temperatures are given by (26)-(27), and for $w$ (31) coupled with (24) give formulae to estimate the coefficient of performance based on two parameters (assuming equal heat conductances on the hot and cold sides, i.e., $\kappa^{*}=1$ ), the ratio of the reservoir temperatures and the relative coefficient of heat leak $\kappa_{L}^{*}$. For the case of vanishing heat leak, the expression approaches the reversible limit. In addition to finite heat transfer rate and heat leak, friction is introduced into the model as a third important source of dissipation. The effect of friction dominates at high speeds, where the maximum $r$ and short circuit points are observed. By including friction, it is possible to explain the general shape of the $r-w$ curves of real refrigeration/heat pump systems. Although the effect of friction dominates at the high-speed limit, its effect in the low-speed limit is negligible, and the derived formulae could be used without any modification.

\section{Acknowledgments}

One of the authors (M. Huleilhil) is very grateful to the Nachemsohn Foundation for financial support and would like to thank the Ørsted Laboratory, University of Copenhagen for further support and hospitality. They would like to thank Professor J. M. Gordon for several enlightening discussions.

\section{References}

[1] R. E. Sonntag and G. J. Van Wylen, Introduction to Thermodynamics, Wiley, New York, NY, USA, 3rd edition, 1991.

[2] H. B. Callen, Thermodynamics and an Introduction to Thermostatics, Wiley, New York, NY, USA, 2nd edition, 1985.

[3] B. Andresen, P. Salamon, and R. S. Berry, "Thermodynamics in finite time," Physics Today, vol. 37, no. 9, pp. 62-70, 1984.
[4] F. L. Curzon and B. Ahlborn, "Efficiency of a Carnot engine at maximum power output," American Journal of Physics, vol. 43, no. 1, pp. 22-24, 1975.

[5] B. Andresen, P. Salamon, and R. S. Berry, "Thermodynamics in finite time: extremals for imperfect heat engines," The Journal of Chemical Physics, vol. 66, no. 4, pp. 1571-1576, 1976.

[6] P. Salamon, A. Nitzan, B. Andresen, and R. S. Berry, "Minimum entropy production and the optimization of heat engines," Physical Review A, vol. 21, no. 6, pp. 2115-2129, 1980.

[7] P. Salamon and R. S. Berry, "Thermodynamic length and dissipated availability," Physical Review Letters, vol. 51, no. 13, pp. 1127-1130, 1983.

[8] M. H. Rubin, "Optimal configuration of a class of irreversible heat engines. I," Physical Review A, vol. 19, no. 3, pp. 12721276, 1979.

[9] P. Salamon, Y. B. Band, and O. Kafri, "Maximum power from a cycling working fluid," Journal of Applied Physics, vol. 53, no. 1, pp. 197-202, 1982.

[10] M. Mozurkewich and R. S. Berry, "Optimal paths for thermodynamic systems: the ideal Otto cycle," Journal of Applied Physics, vol. 53, no. 1, pp. 34-42, 1982.

[11] J. M. Gordon and M. Huleihil, "On optimizing maximumpower heat engines," Journal of Applied Physics, vol. 69, no. 1, pp. 1-7, 1991.

[12] J. M. Gordon and M. Huleihil, "General performance characteristics of real heat engines," Journal of Applied Physics, vol. 72, no. 3, pp. 829-837, 1992.

[13] J. D. Nulton, P. Salamon, and R. K. Pathria, "Carnot-like processes in finite time. I. Theoretical limits," American Journal of Physics, vol. 61, no. 10, pp. 911-916, 1993.

[14] J. D. Nulton, P. Salamon, and R. K. Pathria, "Carnot-like processes in finite time. II. Applications to model cycles," American Journal of Physics, vol. 61, no. 10, pp. 916-924, 1993.

[15] J. Chen and Z. Yan, "Optimal performance of an endoreversible combined refrigeration cycle," Journal of Applied Physics, vol. 63, no. 10, pp. 4795-4798, 1988.

[16] A. Bejan, "Theory of heat transfer-irreversible refrigeration plants," International Journal of Heat and Mass Transfer, vol. 32, no. 9, pp. 1631-1639, 1989.

[17] J. Chen and Z. Yan, "Equivalent combined systems of threeheat-source heat pumps," The Journal of Chemical Physics, vol. 90, no. 9, pp. 4951-4955, 1989.

[18] Z. Yan and J. Chen, "An optimal endoreversible three-heatsource refrigerator," Journal of Applied Physics, vol. 65, no. 1, pp. 1-4, 1989.

[19] J. M. Gordon and K. C. Ng, "Thermodynamic modeling of reciprocating chillers," Journal of Applied Physics, vol. 75, no. 6, pp. 2769-2774, 1994.

[20] P. K. Bhardwaj, S. C. Kaushik, and S. Jain, "Finite time optimization of an endoreversible and irreversible vapour absorption refrigeration system," Energy Conversion and Management, vol. 44, no. 7, pp. 1131-1144, 2003.

[21] Y. Bi, L. Chen, and F. Sun, "Exergetic efficiency optimization for an irreversible heat pump working on reversed Brayton cycle," Pramana, vol. 74, no. 3, pp. 351-363, 2010.

[22] P. K. Bhardwaj, S. C. Kaushik, and S. Jain, "General performance characteristics of an irreversible vapour absorption refrigeration system using finite time thermodynamic approach," International Journal of Thermal Sciences, vol. 44, no. 2, pp. 189-196, 2005.

[23] X. Zhu, L. Chen, F. Sun, and C. Wu, "The optimal performance of a carnot heat pump under the condition of mixed 
heat resistance," Open Systems and Information Dynamics, vol. 9, no. 3, pp. 251-256, 2002.

[24] D. Ladino-Luna and R. T. Paez-Hernandez, "Nonendoreversible Carnot refrigerator at maximum cooling power," Revista Mexicana de Fisica E, vol. 51, pp. 54-58, 2005.

[25] J. Li, L. Chen, and F. Sun, "Fundamental optimal relation of a generalized irreversible Carnot heat pump with complex heat transfer law," Pramana Journal of Physics, vol. 74, no. 2, pp. 219-230, 2010.

[26] C. Petre, M. Feidt, M. Costea, and S. Petrescu, "A model for study and optimization of real-operating refrigeration machines," International Journal of Energy Research, vol. 33, no. 2, pp. 173-179, 2009.

[27] Y. Bi, L. Chen, and F. Sun, "Comparative performance analysis for endoreversible simple air heat pump cycles considering ecological, exergetic efficiency and heating load objectives," International Journal of Exergy, vol. 6, no. 4, pp. 550-566, 2009.

[28] Y. Ust and B. Sahin, "Performance optimization of irreversible refrigerators based on a new thermo-ecological criterion," International Journal of Refrigeration, vol. 30, no. 3, pp. 527534, 2007.

[29] L. Chen, J. Zheng, F. Sun, and C. Wu, "Power density optimization for an irreversible closed brayton cycle," Open Systems and Information Dynamics, vol. 8, no. 3, pp. 241-260, 2001.

[30] B. Sahin and A. Kodal, "Thermoeconomic optimization of a two stage combined refrigeration system: a finite-time approach," International Journal of Refrigeration, vol. 25, no. 7, pp. 872-877, 2002.

[31] K. Ma, L. Chen, and F. Sun, "Profit rate performance optimization for a generalized irreversible combined refrigeration cycle," Sadhana, vol. 34, no. 5, pp. 851-864, 2009.

[32] ASHRAE Handbook, Fundamentals, American Society of Heating, Refrigeration and Air Conditioning Engineering, 1977.

[33] F. Sisto, "The reversed Brayton cycle heat pump-a natural open cycle for HAVAC applications," Journal of Engineering for Power, vol. 101, no. 1, pp. 162-167, 1979.

[34] M. O. McLinden, "Thermodynamic evaluation of refrigerants in the vapour compression cycle using reduced properties," International Journal of Refrigeration, vol. 11, no. 3, pp. 134$143,1988$. 

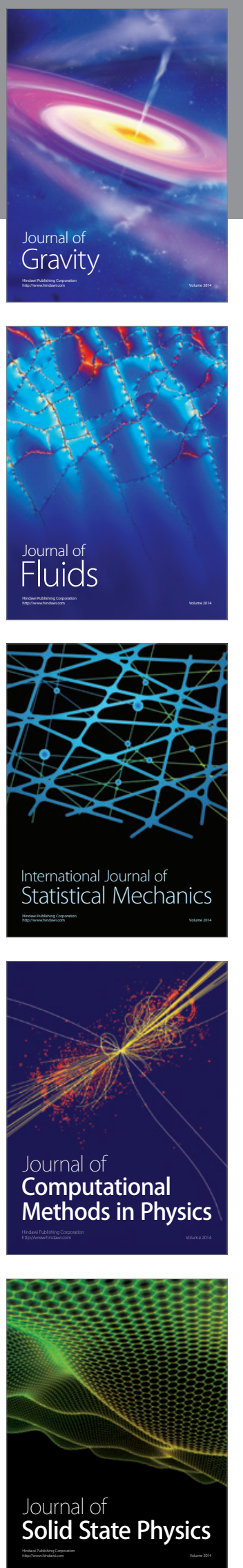

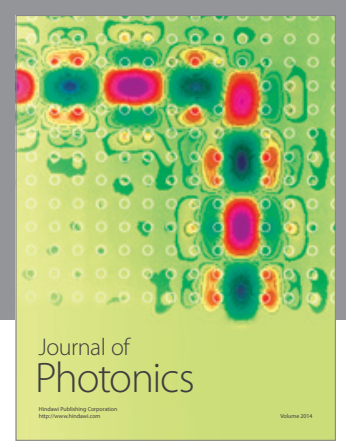

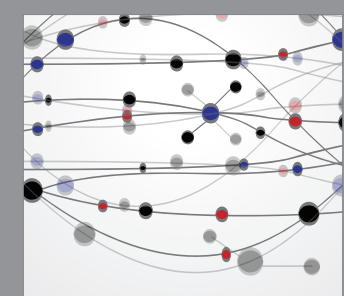

The Scientific World Journal
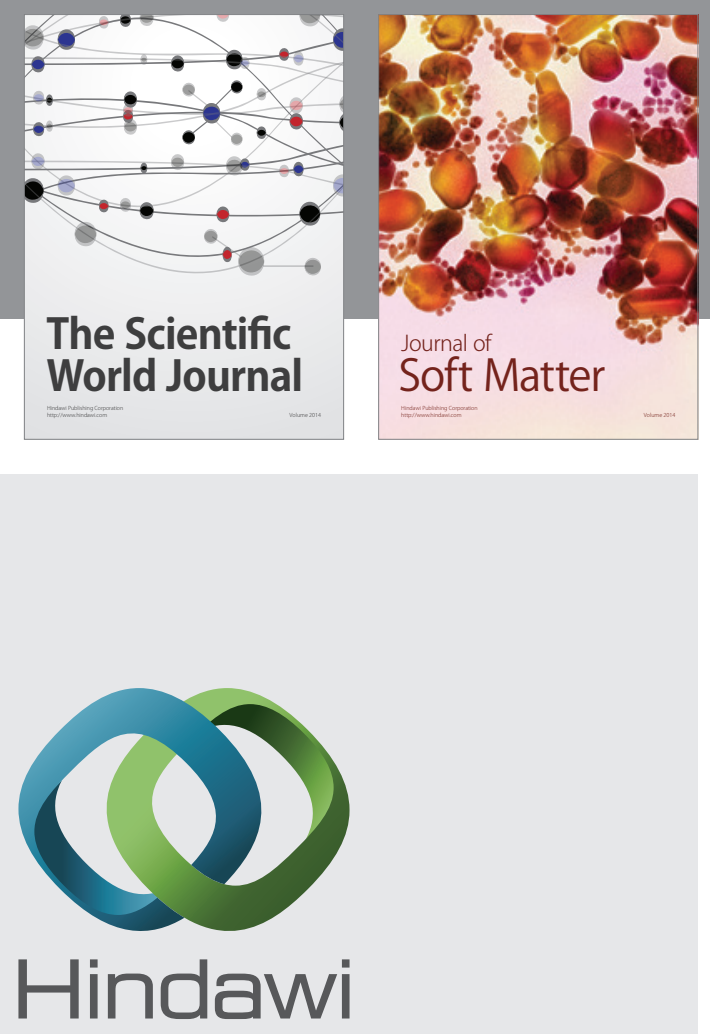

Submit your manuscripts at

http://www.hindawi.com
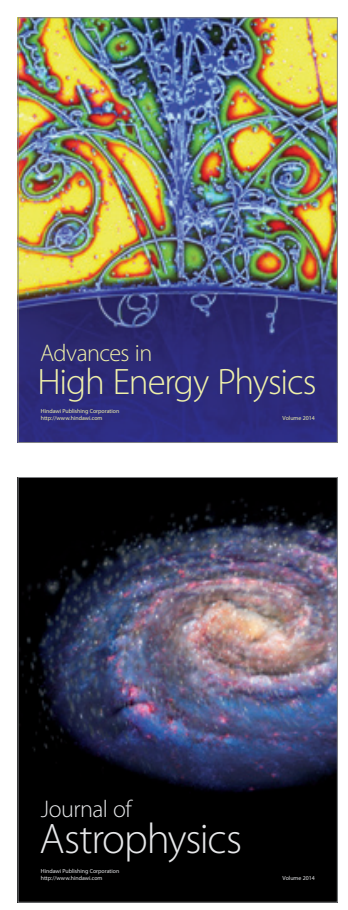
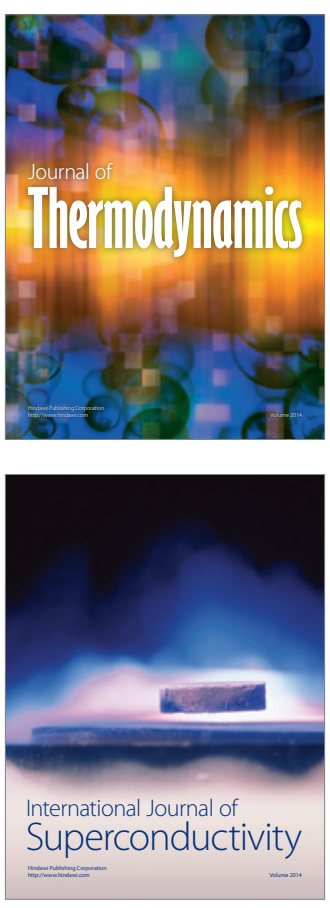
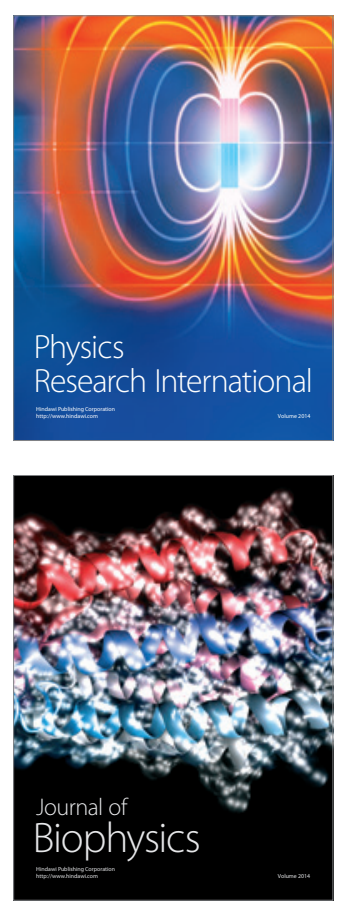
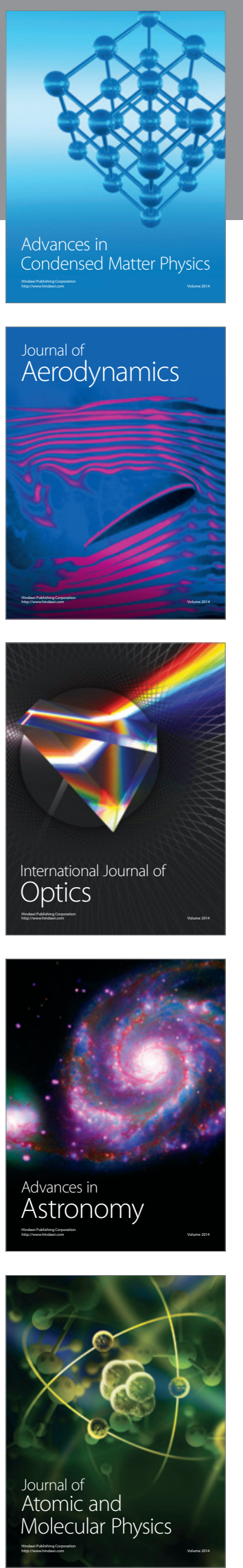